\title{
Reimbursement categories as a way of allocating measures and monitoring expenditures on medicines
}

\author{
Aneta Lipińska*, Maciej Pomorski, Wojciech Matusewicz, Katarzyna Jagodzińska-Kalinowska \\ From 3rd International PPRI Conference 2015: Pharmaceutical Pricing and Reimbursement Policies: Challenges \\ Beyond the Financial Crisis \\ Vienna, Austria. 12-13 October 2015
}

\section{Background}

Control of expenditure on reimbursement of medicines distributed to patients through pharmacies poses many problems despite it providing easy access to the drugs. Other reimbursement categories, chemotherapy drugs and drug programs, may allow health authorities to allocate measures and monitor expenditures under the whole budget for drugs.

\section{Objectives}

The aim of this publication is to describe two reimbursement categories in Poland, chemotherapy drugs and drug programs, and to assess strengths and weaknesses of them from the payer's and patients' perspective.

Policies targeted: The abstract presents evaluation of reimbursement categories which interface between outpatient and in-patient sectors, and influence of these reimbursement categories on public payer, health care providers (HCP), and patients.

\section{Methods}

This work is a description of a model implemented in Poland and an evaluation of current (up to 28 May 2015) policy in terms of rationalizing and controlling expenditures and access to health care services. The study examines the public sector and is related to the out-patient as well as in-patient sectors because of the mixed nature of these solutions.

\section{Results}

The essence of the solution implemented in Poland is agreements between the payer (National Health Fund

\footnotetext{
* Correspondence: a.lipinska@aotm.gov.pl

Agency for Health Technology Assessment and Tariff System, Warsaw, 02611, Poland
}

(NHF)) and individual providers, but what is important is the NHF nor HCP are obligated to enter into drugs are distributed by ho basis. Their consumption is reported in detail to the NHF. Drug programs ensure that the qualifying criteria are the same, the monitoring of therapy effects is simplified and the NHF can control if it pays for the successful treatment. of services and maximum expenditures. On the other hand, adherence to the limit and freedom in concluding contracts may restrict the availability of services (queues, migration of patients in search of treatment) and burden of therapy may harm patients more than unsuccessful

\section{Conclusions}

categories limits access - only through contracted HCP (hospital and ambulatory care), but on the other hand it rationalizes the use of medicines. Careful analysis of health allow a proper allocation of resources and ensuring patients an equal access to services.

Published: 5 October 2015 